\author{
Military Technical College \\ Kobry Elkobbah, \\ Cairo, Egypt
}

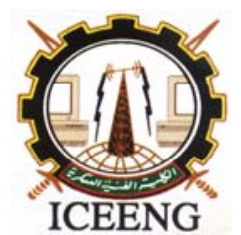

\title{
MEASURING DEPTH OF ANESTHESIA USING BISPECTRAL ANALYSIS OF THE ELECTROENCEPHALOGRAM (EEG)
}

\author{
Essam* A., M. ** A. Elkfafi, S. *** Alian, M.***. E. Gadallha
}

\begin{abstract}
The assessment of patient brain state during surgery has long been an objective of research in the field of automated electroencephalogram (EEG), since EEG signals contain a wealth of information about brain functions, there are many approaches to analyzing EEG signals with spectral techniques. In this paper the bispectral analysis was applied to EEG signals obtained from patients under general anesthesia by using BSA software (bispectrum analyzer software version 3.31B) [14]. The bispectral values (BIS) was normalized to 100 while awake and decreasing gradually to a level between 0 and20 as the patient was anaesthetized. The clinical signs such as heart rate (HR) and blood pressure (BP) of the level of anesthesia were observed simultaneously. Conclusion, the bispectral analysis of EEG signals obtained from patients under general anesthesia gives accurate index to measure depth of anesthesia.
\end{abstract}

\section{KEY WORDS}

Electroencephalogram, depth of anesthesia, bispectral analysis, sedation, BIS index.

\section{Introduction}

Clinicians administering anesthetics and sedatives need to manage the hypnotic state of their patients. Hypnosis is defined as the impairment of consciousness and memory. Patients undergoing surgery require an adequate level of hypnosis to protect them from stress and awareness; it is not currently possible, however to directly measure the hypnotic state. At lighter levels an indirect assessment of the hypnotist state is performed clinically by observing physical signs and patient responsiveness to voice or touch. Although this approach is adequate in some situations, it has several significant limitations. Assessment using patient responsiveness is not applicable to patients who are incapable of responding[5].

In response to these limitations, researchers have sought an object measurement of the hypnotic state that can be acquired continuously without disturbing the patient. Because the EEG shows changes with hypnotic state. Scientists have examined ways to automate EEG analysis to create a measure that is indicative of these changes. This paper describes the bispectral analysis of EEG signals gives adequate index to measure the changes of hypnotic state[6].

* Graduate student, Biomedical Department, MTC, Cairo, Egypt.

** PHD, Electronic Department, MTC, Cairo, Egypt.

*** Professor, Electronic Department, Modern Academy, Cairo, Egypt.

*** Professor, Biomedical Department, MTC, Cairo, Egypt.

\section{Changes in EEG with increasing sedation}


EEG sedation measures have been based on the observation. That EEG generally changes from low amplitude, high frequency signal while awake to large amplitude, low frequency signal while deeply anaesthetized. The general idealized pattern of changes that occur in the EEG as the level of hypnosis is increased is shown in Figure (1) these changes maybe described using frequency bands in table (1) the EEG signals can be bracken down into a series of sine wave components such that when the components are combined together, the original signal is recovered. The decomposition of an EEG signal into it is components are typically performed to characterize the signal by a single number or set of numbers that are easily trended over time. These numbers are referred to as processed parameters. Most EEG parameters used today based on power spectral analysis (second order statistics) which represents the amplitudes (or power) of each sine wave components as a function of frequency. One commonly used descriptor that attempts to track the changes that occur with increasing level of hypnosis is the 95\% spectral edge frequency (SEF) [7-8]. The 95\% SEF is the frequency below which the power spectrum contains $95 \%$ of the total power in the EEG signal. It is an adequate measure of sedation state in some limited situations however the $95 \% \mathrm{SEF}$ becomes less useful.

Table 1. Classification of the EEG based on the dominant frequency

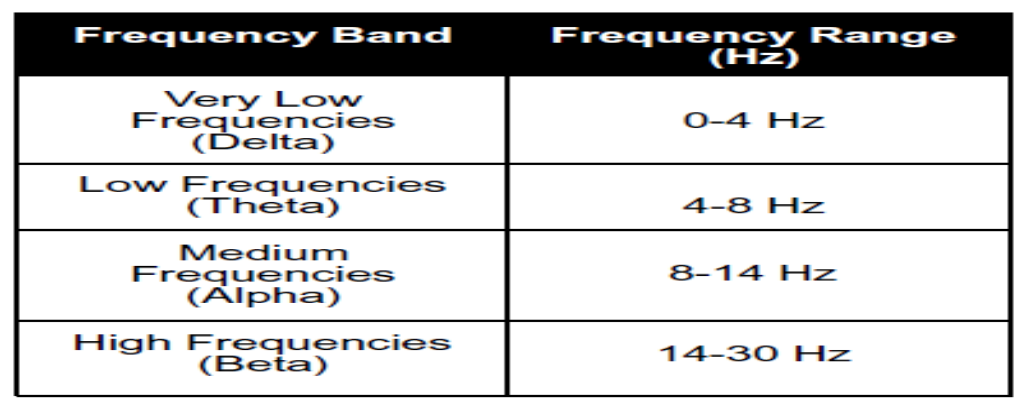
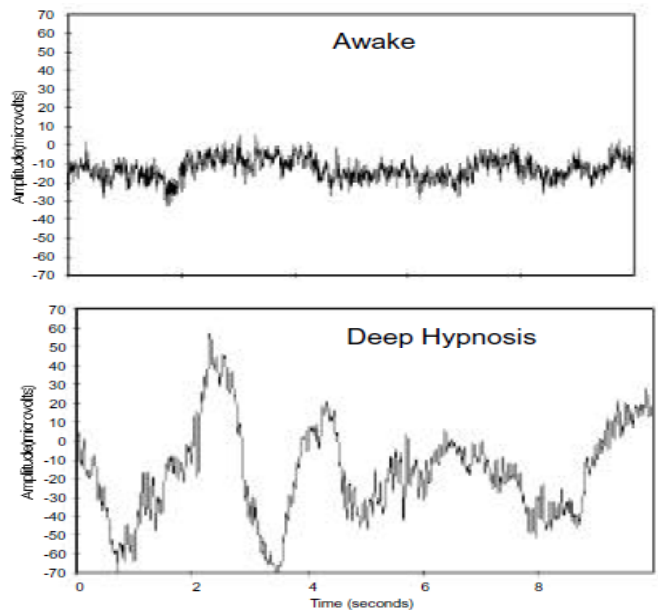
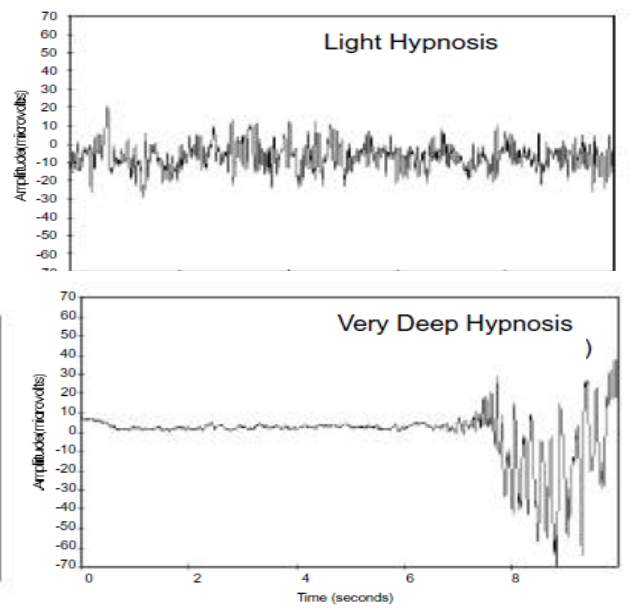

Fig.1. General, idealized pattern of changes in the EEG as a level of hypnosis increases

When the EEG signal does not transition smoothing from high frequencies to low frequencies as anesthetic dose increases or the different frequencies with in the EEG signal may not be independent of each other. if the oscillations are linked by a common phase relationship, so it is necessary to use third order statistics to extract this information. Bispectral analysis is a method of analysis which examines the relationship or phase coupling between different frequencies bispectral 
analysis quantifies the level of synchronization in the EEG with traditional amplitude and frequency parameters [9-10].

\section{Methods}

Patient data and EEG signal acquisition

We studied 4 patients ( 3 females mean age $=41$ years) under going elective surgery. No patients had a history of neurological disorder. The patients received midazolam 1-3 mg and fentanyl 50-100 ug i.v followed by induction of anesthesia with propofol $80-200 \mathrm{mg}$ i.v. the clinical anesthetist was blinded to the EEG monitoring. Electrode skin impedance was reduced to $<5000 \Omega$ by cleaning the skin with an abrasive cleaning fluid and using a low impedance electrode paste. We used disposable adhesive silver/silver chloride electrodes. The EEG was recorded using bipolar bifrontal montage $(\mathrm{F} 7-\mathrm{F} 8)$ with the ground electrode placed in the mid forehead position $(\mathrm{Fz})$ the recording raw EEG signal (sampling frequency $128 \mathrm{~Hz}$ ) were band-pass filtered to 0.5 to $30 \mathrm{~Hz}$ and processed in real time using BSA software of the BIS algorithm, additional quantitative EEG variables including $95 \%$ SEF were also calculated on line. With the help of serial port the EEG variables were digitally recorded every $30 \mathrm{sec}$.for the duration of the study. Data were stored on a personal computer as a text file and analyzed offline with the helpful Microsoft Excel.

\section{Results from clinical validation studies}

From the literature review described before we are satisfied that the EEG shows promise for measuring the DOA. To clinically evaluate the bispectral variables derived from the EEG data were recorded from 4 patients, who were undergoing body surface surgery, during intravenous anesthesia via propofol At the same time, the propofol concentration (PC) was measured one minute before stopping of propofol and every minute after the stopping of propofol (i.e. during the recovery phase). The EEG data corresponding to the period of measuring the PC were selected from the EEG data files of the 4 patients. These EEG data were processed using the digital signal processing technique as mentioned above. The numerical index (bispectral values) were used to facilitate racking the changes in the EEG according to the changes in propofol concentration during time recovery phase. The index and PC were plotted versus the recovery time as shown in figure (2). The changes in the above factor, with different PC, during the recovery phase were examined. It was found that, the bispectral values were increasing when the PC values were decreasing. This result confirms that the bispectral index detected the awareness (i.e. eye opening) successfully as shown in Figure (2) and the anesthetist's experiments for detecting the awareness using the clinical signs. Figure (3) represents the BIS index, heart rate (HR) and blood pressure (BP) for patient who were undergoing body surface surgery, during intravenous anesthesia via propofol
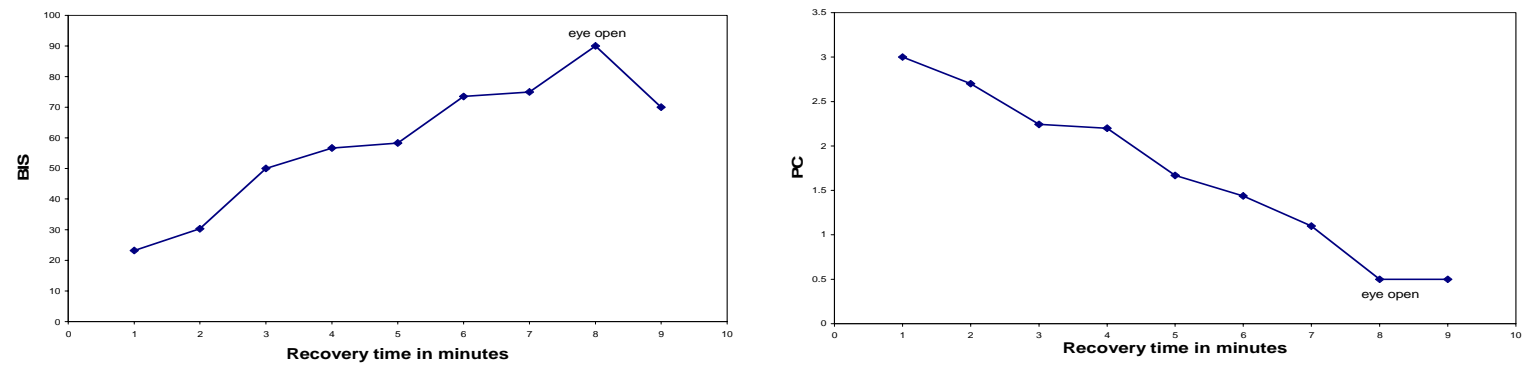
Fig.2. The changes of bispectral index and PC every one minute during the recovery phase of the patient

\section{Conclusion}

The Bispectral Index and the hypnotic effects provided by propofol have also recently been described in a number of studies. These studies concluded that the BIS provide a quantifiable measure of the effects of anesthetics on the brain that correlates to the level of consciousness and probability of recall. BIS was also found to be useful as an indication of return of consciousness during general anesthesia.

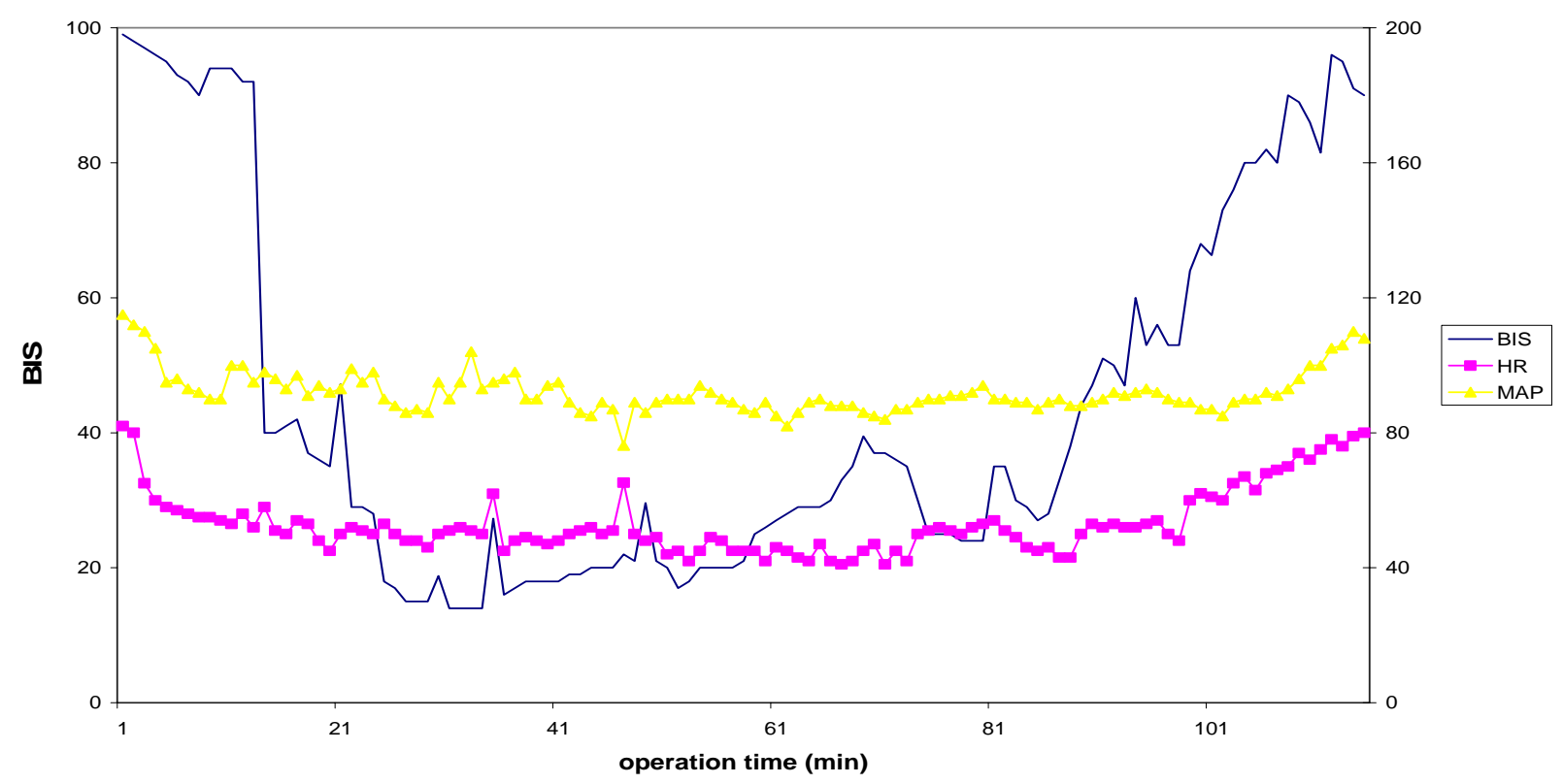

Fig.3. the BIS index, heart rate (HR) and blood pressure (BP

\section{REFERENCES}

[1] Flaishon, R., Sigal "Bispectral analysis of the EEG for monitoring the Hypnotic effect of propofol/Aflentanil" Anesthesiology, vol.83, No.3A, A514, (1995).

[2] Boolm, M. et.al."Bispectral index as an EEG measure of sedation" Anesthesiology, vol.85 No.3A, A461, (1996).

[3] Liu, J., Harbhej, S., "Electroencephalogram Bispectral analysis predicts the depth of Midazolam-induced sedation” Anesthesiology, vol.84, No.1, pp64-69, (1996).

[4] Satosh Hagihira "Electroencephalographic Bicoherence is sensitive to noxious stimuli during Isoflurane or Sevoflurane anesthesia” Anesthesiology, 100(4), 818-825 (2004).

[5] Rampil IJ "A primer for EEG signal processing in anesthesia” Anesthesiology, 98(4), 890-1002 (1998).

[6] Goto T "Depth of anesthesia monitored with bispectral index" (2001).

[7] Scott D. Kelley "Monitoring level of consciousness during anesthesia and sedation", A clinician guide to the bispectral index (2003).

[8] Physiometrix "The brain the organ of consciousness, use of processed EEG to monitor the effect of anesthetic agents" (2001). 
[9] Katoh T.,Bito H.,'Influence of age on hypnotic requirement,bispectral index, and 95\% spectral edge frequency associated with sedation induced by sevoflurane" Anesthesiology, 92:19-25, (2000). [10] Nieuwenhuijs D., "Bispectral index values and spectral edge frequency at different stages of physiologic sleep" Anesthesiology, 94:125-129, (2002). 
